\title{
Towards a versatile European Driver Advisory System
}

\author{
D. Emery \\ École Polytechnique Fédérale de Lausanne (EPFL), Switzerland
}

\begin{abstract}
Pacing trains is an important part of railway operations management. It helps to improve the resilience of a timetable to perturbation and reduces significantly both the energy consumption and the wear-and-tear, while increasing the comfort for passengers. Today, only a few Driver Advisory Systems (DAS) with such abilities are already in operation on European major railways. This situation provides great opportunity to promote a unique but flexible system, which can be implemented and used around Europe.

The paper describes a concept for a Versatile Driver Advisory System (VDAS). The on-board part of this system is not only fully compatible with a European Driver Advisory System (EDAS) coming from Traffic Management Systems (TMS) but also with other DAS (NEDAS). To ensure flexibility and versatility, EDAS can be implemented in different ways. The use of the standard railway radio GSM-R and the opportunity to send speed advices on the ETCSDMI are milestones on the development of ETML, the management layer of ERTMS.

VDAS facilitates the improvement of the operational resilience to perturbation as well as sparing energy, offers synergy opportunities with the ETCS On-board system and, finally, VDAS is based on EDAS which may become a European standard.
\end{abstract}

Keywords: traffic flow, free-flow, capacity management, real time traffic management, speed advice, energy-saving, DAS, ERTMS.

\section{Introduction}

Sustainable development has gained more and more importance over the past decades. Even if railways are already an energy-efficient mode of transport, 
many Driver Advisory Systems (DAS) have recently been designed to minimise the energy consumption of trains.

Most of them are stand-alone DAS. Such systems compare the planned timetable of the train with the actual time and position often given by a GPS device. DAS displays speed recommendations to drivers according to its more or less full knowledge of time allowances (performance margins) added to the minimal travel time on one side, and of the train and track characteristics and static maximal speed profile on the other side. In case an unexpected speed restriction appears, such a system is unable to display advice minimising the global delay in this sub-network. Furthermore, it is unable to compute and display speed advice to minimize the train's own delay. Finally, even the energy minimisation goal is compromised by the lack of knowledge concerning the position and speed of others trains.

Often, congested railway sub-networks are more interested in capacity management than in energy consumption. By chance, in many cases, energy savings and optimal capacity management are positively related.

That's why some Infrastructure Managers (IMs) are developing their own DAS in tight relation with their Traffic Management Systems [1].

\section{A Versatile European Driver Advisory System (VDAS)}

It is urgent to develop DAS standards at the European level (cf. a very first attempt in [2]). Then it will be possible to avoid repeating the dead-lock situation ETCS has had to solve (too many different ATP systems and at least three different cab signalling systems for high speed, cf. [3]). The European ON-TIME project, in which are involved many major European IMs (NR_UK, RFI_IT, DB_DE and RFF/SNCF_FR) has probably a central role to promote a European Driver Advisory System (EDAS) (cf. [4, 5]).

The aim of the Versatile Driver Advisory System (VDAS), introduced here, is dual. First, VDAS would promote EDAS, in synergy with all three ERTMS components: GSM-R, ETCS and ETML. Secondly, VDAS would allow continuing to use present DAS systems (cf. figure 1).

\subsection{EDAS tasks and their share out between EDAS-TMS and EDAS-Train}

According to the European ON-TIME project works, three main tasks have to be fulfilled by EDAS. The first task is to compute successive advice speed targets to be respected at specific positions (speed range/time range/position). The last target is a fixed speed/time/position triplet. The ON-TIME project uses the term "train path envelope" to describe the highest top-speed and lowest bottom-speed trajectories of the train capable to reach one speed target from the previous one, respecting physical and legal laws. The second task is to compute an optimal speed profile according to criteria such as comfort of passengers, energy consumption, and convenience to translate the optimal speed profile into guidance and then displayed advice. The third and last main task is to compute the advice itself. 


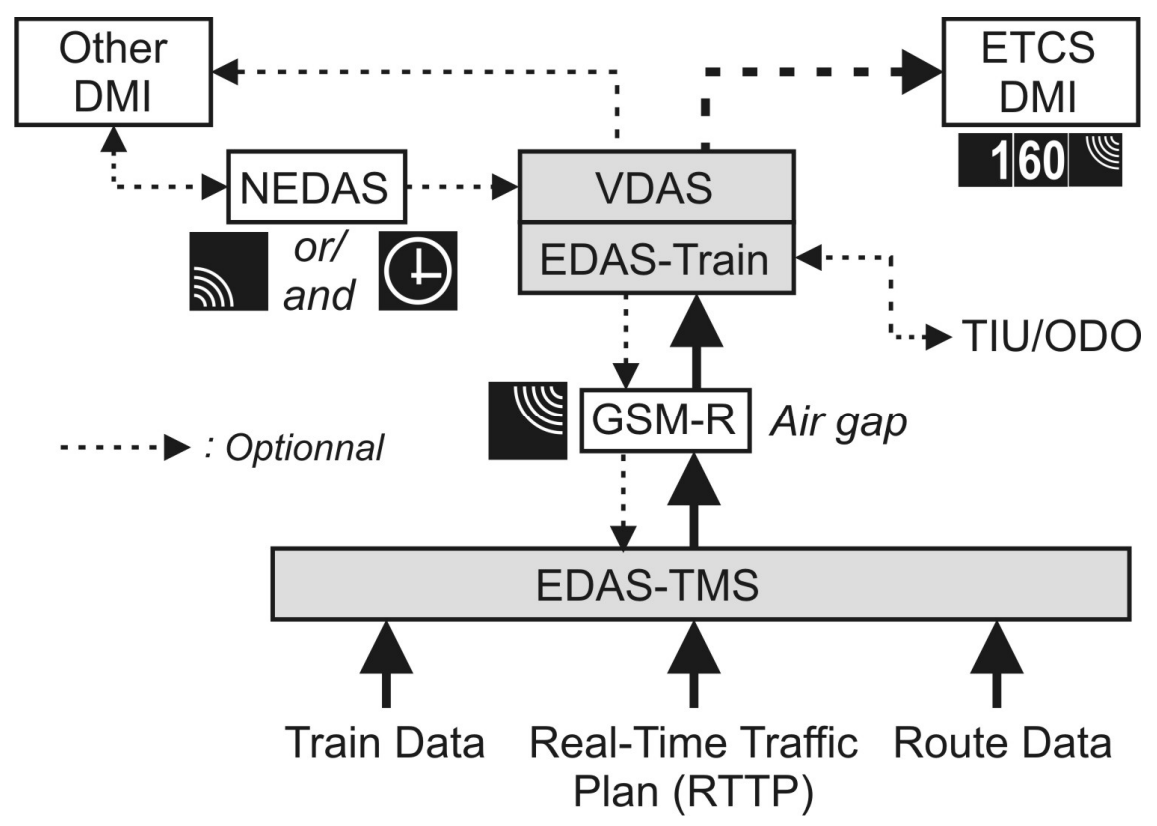

Figure 1: Functional block diagram of a Versatile Driver Advisory System (VDAS).

The first task must be done only by TMS, as speed targets are highly dependent on position and speed of the others trains.

The second as well as the third task can be done either by EDAS-TMS or by EDAS-Train (cf. figure 2).

In the proposed EDAS system, the layout is not set yet but DAS-C and DASO have some advantages versus DAS-I (see figure 2), regarding in particular the data flow through the GSM-R radio and the simplicity of the displayed advice on the ETCS-DMI (see figure 1). The assumption is made that DAS-I will be discarded in EDAS.

\subsection{VDAS and Not EDAS (NEDAS)}

The versatility of VDAS is reinforced by the bi-directional link between VDAS and NEDAS. In one direction VDAS offers the opportunity to actual NEDAS systems to have access to the ETCS-DMI, in particular for areas not fit with EDAS-TMS. The main advantage is that drivers always find speed advice at the same location in the cab, just under the speed main display they frequently look at. In the other direction, NEDAS-DMI offers the opportunity to EDAS to display speed advice even if the cab is not equipped with the ETCS-DMI display (cf. figure 1). 


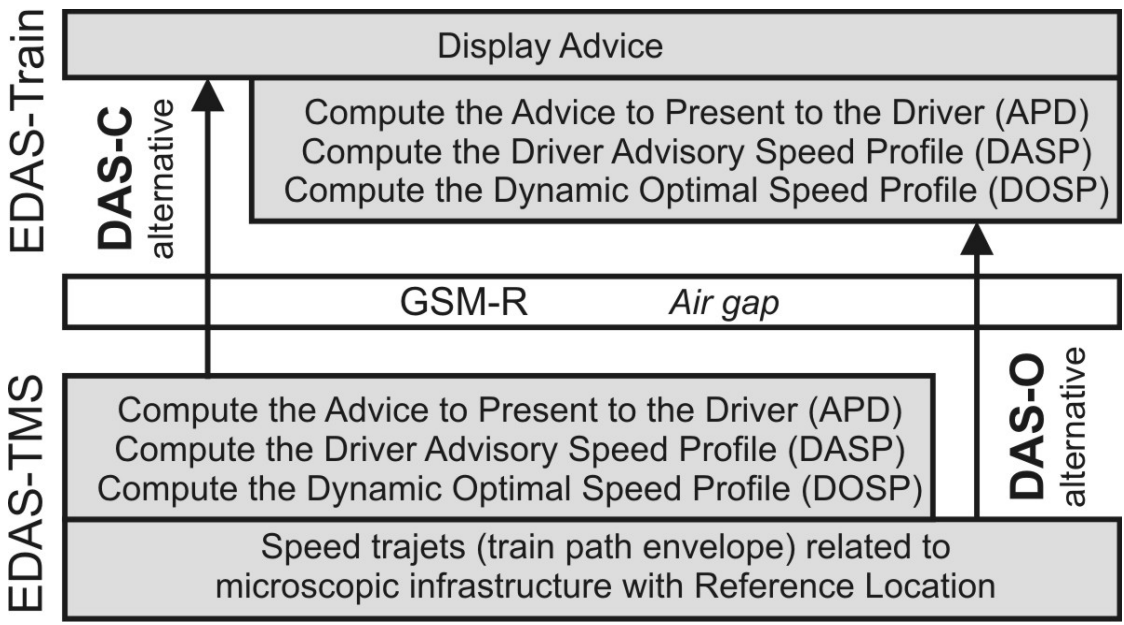

Figure 2: Alternatives DAS-C and DAS-O for sharing DAS tasks between TMS and train, according to the European ON-TIME project (cf. [5]).

\subsection{VDAS modes and states}

VDAS has four different modes (called levels according to the ETCS vocabulary, cf. [6], [7]): EDAS-O (O), EDAS-C (C) NEDAS (N) and Unfitted (U). The table 1 shows the possible and recommended modes according to the fitting of EDAS-TMS and VDAS-Train.

Table 1: $\quad$ Possible and recommended (bold) modes for VDAS.

\begin{tabular}{|l|c|c|c|}
\hline VDAS-TRAIN: & \multicolumn{5}{|l|}{} \\
\hline$E D A S-O+N E D A S$ & $\mathrm{~N}$ & $\mathrm{~N}, \mathbf{O}$ & $\mathrm{N}, \mathrm{C}, \mathbf{O}$ \\
\hline$E D A S-O$ & $\mathrm{U}$ & $\mathrm{O}$ & $\mathrm{C}, \mathbf{O}$ \\
\hline$E D A S-C+N E D A S$ & $\mathrm{~N}$ & $\mathrm{~N}$ & $\mathrm{~N}, \mathbf{C}$ \\
\hline$E D A S-C$ & $\mathrm{U}$ & $\mathrm{U}$ & $\mathrm{C}$ \\
\hline$N E D A S$ only & $\mathrm{N}$ & $\mathrm{N}$ & $\mathrm{N}$ \\
\hline$E D A S-T M S:$ & Unfitted & $E D A S-O$ & $E D A S-C$ \\
\hline
\end{tabular}

VDAS has seven possible states (called modes according to the ETCS vocabulary, cf. [6], [7]): speed advice from EDAS-O (EO), from EDAS-C (EC), from NEDAS using Radio communications (NR), from NEDAS based on a planned Timetable (NT), Stand-By (SB), System Failure (SF) and No Power (NP).

Transitions between states are given by a transition table. No direct transitions between speed advice modes $(\mathrm{O}, \mathrm{C}$ and $\mathrm{N})$ are allowed: they must pass through the Stand-By mode. 


\subsection{EDAS-TMS}

The main compulsory task of the EDAS-TMS is to compute speed targets (cf. §.2.1). A group of targets must contain reference locations (RL), time stamps, distances from RL, expected time and speed at target location, as well as tolerance intervals. According to the train and route characteristics, targets must be compatible with the Most Restrictive Dynamic Speed Profile (MRDSP) of the train. MRDSP may be considered similar to an ETCS Permitted Speed Profile.

\subsection{Computing the optimal speed profile and the speed advice profile}

The computing of the Dynamic Optimal Speed Profile (DOSP) and the computing of the Driver Advisory Speed Profile (DASP) may be performed in the trackside EDAS-TMS module (EDAS-C) or in the on-board EDAS-Train module (EDAS-O). DOSP is the speed/distance curve that a train may follow to optimise the real-time capacity management. DASP is the reduction of DOSP so the Advice to Present to the Driver (APD) shall not change too often. DOSP, as well as DASP, shall not be higher than the MRDSP.

EDAS Product design could have a relatively low Safety Integrity Level (SIL) if APD disappears surely and immediately in case of the arrival of the colour orange or red on the DMI (see table 3).

\subsection{Speed advice displayed on ETCS-DMI and on another DMI}

Since speed advice is information not directly related to safety, ADP has to be placed in the Supplementary Driving area (C) of the ETCS-DMI and not in the Speed info area (B) of the DMI. Three main reasons strengthen this choice. Firstly, Speed Advice is clearly separate from actual speed and LoA. Secondly, all available areas in B (B6, B3/B4/B5, B7) can already be occupied: B6 for release speed, $\mathrm{B} 3 / \mathrm{B} 4 / \mathrm{B} 5$ for track conditions and $\mathrm{B} 7$ for ETCS active

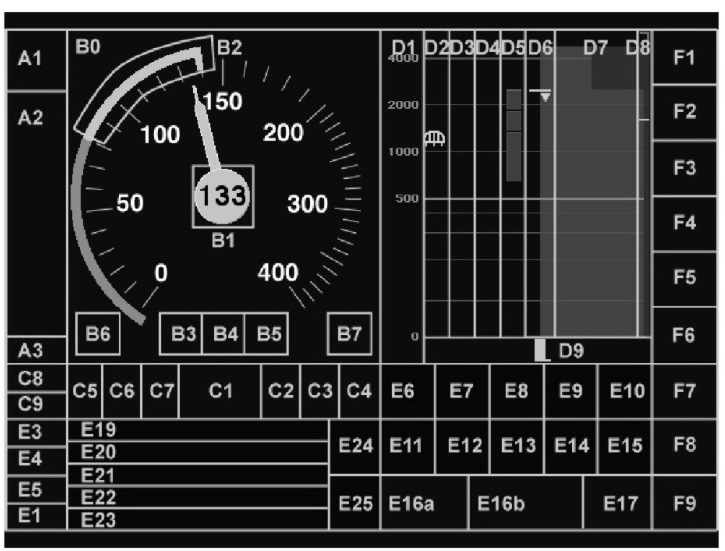

Figure 3: ETCS-DMI main areas [8]. 
mode (cf. [8]). The last but not least reason is that the ERTMS Operational Rules Group has already proposed to use C2, C3 and C4 for speed advice purposes $[10]$.

\subsubsection{Advice to present to the driver on the ETCS-DMI}

The Advice to Present to the Driver (ADP) is only a speed advice given with one to three digits from 0 to the maximal speed and a symbol indicating the origin of the advice. Use of a symbol indicating the ADP origin and a simultaneous coasting recommendation is still an open issue.

Nowadays, the optimal free flow regarding energy and capacity consumptions can be realised only if the driver is informed well in advance of a potential conflict with another train. In most of the cases, the advice will primarily consist of a proposal made to the driver to set the speed significantly lower than the permitted speed. It is then relevant to propose a gradually attained speed advice. Experienced drivers, having a good knowledge of the line characteristics and the train properties, can decide to reduce gradually the speed of the train with the appropriate means (e.g. coasting phases, electrical braking only).

Even for a speed advice higher than the actual speed, the gradually attained speed advice gives the driver the opportunity to wait for a steep downslope that will accelerate the train.

The rapidly attained speed advice suggests hurrying up to reach the indicated value. To slow down the train, drivers are invited to decelerate the train with about half the deceleration of a braking-to-stop. Deceleration is easy to be determined by experienced drivers who know perfectly the brake percentage of their train and the track gradient. The rate of deceleration is not fixed yet. Maybe the rapidly attained speed advice would have to include two different options: rapidly attained speed advice and very rapidly attained speed advice. This last advice may lead to full braking. If acceleration is required, full power is requested.

A lot of different alternatives are possible to display APD. The use of different colours is foreseeable as well as sounds. Flashing of frames and highlighting is acceptable (see [8]).

Concerning sounds, ETCS-DMI can provide four different sounds that are the same for all of Europe: "click", "S_info", S1_toofast" and "S2_warning" [8]. The "click" sound is used only to give a feedback while pressing the finger on a button and S1 and S2 each have a very specific meaning. They therefore cannot be used by DAS. To avoid overloading the drivers with sounds, it is proposed to use S_info only when VDAS enters EO, EC or NR or NT states. No sound is also an alternative. In that case, flashing the first ADP displayed seems convenient.

The orange and the red colours have a very specific meaning and are therefore forbidden for displaying speed advices. The use of the yellow colour for ADP is still an open issue. As long as something is displayed in orange or in red in the DMI, no APD must be displayed. If an APD is being displayed when something appears in orange or red, APD must be switched off immediately (cf. figure 4). 
Whether APD should stay when the main speed display shows something in yellow is still an open issue.

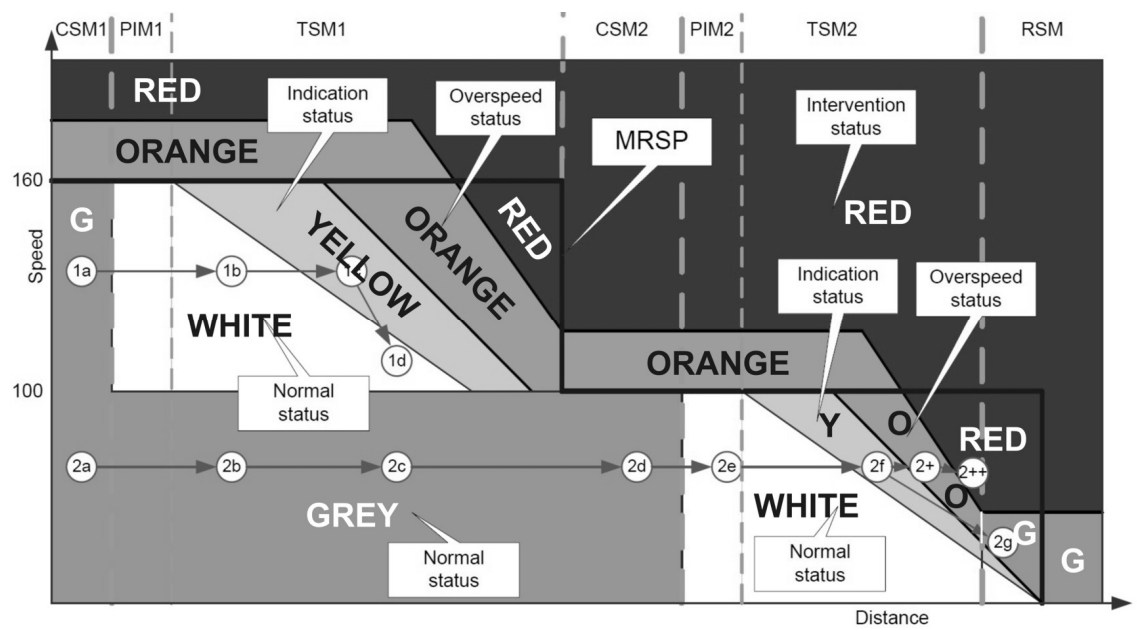

Figure 4: Principles of use of colours for the main speed display of the ETCS-DMI ([8] and [9]).

Table 3: Some APD possible aspect alternatives.

\begin{tabular}{|c|c|c|c|}
\hline APD & $\begin{array}{c}\text { Alternative } \\
\text { "grey_shade" }\end{array}$ & $\begin{array}{c}\text { Alternative } \\
\text { "grey high" }\end{array}$ & $\begin{array}{c}\text { Alternative } \\
\text { "grey-white" }\end{array}$ \\
\hline no & (background) & (background) & (background) \\
\hline $\begin{array}{c}\text { Gradually } \\
\text { attained } \\
\text { speed }\end{array}$ & $\begin{array}{c}\text { Steady } \\
\text { Medium } \\
\text { Grey }\end{array}$ & $\begin{array}{c}\text { Steady } \\
\text { Medium } \\
\text { Grey }\end{array}$ & $\begin{array}{c}\text { Steady } \\
\text { Grey }\end{array}$ \\
\hline $\begin{array}{c}\text { Rapidly } \\
\text { attained } \\
\text { speed }\end{array}$ & $\begin{array}{c}\text { Steady } \\
\text { Grey }\end{array}$ & $\begin{array}{c}\text { Highlight } \\
\text { Grey }\end{array}$ & $\begin{array}{c}\text { Steady } \\
\text { White }\end{array}$ \\
\hline $\begin{array}{c}\text { Speed to } \\
\text { maintain }\end{array}$ & $\begin{array}{c}\text { Steady } \\
\text { Dark }\end{array}$ & $\begin{array}{c}\text { Steady } \\
\text { Dark } \\
\text { Grey }\end{array}$ & $\begin{array}{c}\text { Steady } \\
\text { Medium } \\
\text { Grey }\end{array}$ \\
\hline
\end{tabular}

Usable colours are defined in [8]. For ex. Dark Grey is Red-85/Green85/Blue-85, Grey 195/195/195 and White 255/255/255.

A coasting advice may be added to the list of APD aspect. It may be given with or without a speed target. 


\subsubsection{Transitions between advice presented on the ETCS-DMI}

(1) Only if the newly maintain ADP displayed is close to the actual speed of the train and sound $\mathrm{S}$ info or frame flashing is provided.

(2) In case of the orange/red colour arrival on DMI, or EDAS enter the NP, $\mathrm{SB}, \mathrm{SF}$ or UN state.

(3) In case of deceleration, only if the new proposed speed is lower than the actual speed. In case of acceleration, only if the new proposed speed is higher than the actual speed.

The minimum time, during which a certain APD must be displayed before being updated, is not set yet.

Table 1: APD transitions.

\begin{tabular}{|l|c|c|c|c|}
\hline $\begin{array}{l}\Gamma \longrightarrow \text { to } \\
\text { from }\end{array}$ & nil & $\begin{array}{c}\text { gradually } \\
\text { attained }\end{array}$ & $\begin{array}{c}\text { rapidly } \\
\text { attained }\end{array}$ & maintain \\
\hline nil & & Yes & Yes & Yes (1) \\
\hline gradually attained & Yes (2) & Yes & Yes & Yes \\
\hline rapidly attained & Yes (2) & Yes & Yes (3) & Yes \\
\hline maintain & Yes (2) & Yes & Yes & Yes (1) \\
\hline
\end{tabular}

\subsubsection{Advices presented on another display}

The Speed Advice provided by EDAS can also take place on the GSM-R-DMI, on a NEDAS-DMI or on a specific display. It is conceivable to complete the advice with a guidance speed curve [4].

\section{Conclusion}

A Versatile European Driver Advisory System (VDAS) offers three main advantages.

First, VDAS facilitates the improvement of the operational resilience to perturbations as well as saving energy, not only for the area equipped with EDAS but also thanks to other DAS.

Secondly, VDAS offers synergy opportunities with the ETCS On-board system. Driver advisory may take place on the ETCS-DMI and train data can be shared through the Train Interface Unit (TIU). In particular, it could be of great advantage to benefit from the results of the ODOmetry unit (ODO), and from the static train data as well. Thanks to the progress made within the On-Time European project, EDAS may become a European standard.

Finally, the possibility of a step-by-step introduction of the new technology may help manufacturers, railway companies and infrastructure managers to implement compatible DAS in trains and in traffic management systems. 


\section{Abbreviations and acronyms}

\begin{tabular}{|c|c|}
\hline APD: & Advice to Present to the Driver \\
\hline ATP: & Automatic Train Protection system \\
\hline BACC: & Blocco Automatico a Correnti Codificate \\
\hline DA: & Driver Advisory state \\
\hline DASP: & Driver Advisory Speed Profile \\
\hline DMI: & Driver Machine Interface \\
\hline DOSP: & Dynamic Optimal Speed Profile \\
\hline EDAS: & European Driver Advisory System \\
\hline EEIG: & European Economic Interest Group \\
\hline ERTMS: & European Rail Traffic Management System \\
\hline ETCS: & European Train Control System \\
\hline ETML: & European Traffic Management Layout \\
\hline FRS: & Functional Requirements Specification \\
\hline GSM-R: & Global System for Mobile Communications - Railways \\
\hline GPS: & Global Positioning System \\
\hline IM: & Infrastructure Manager \\
\hline LoA: & Limit of Authority \\
\hline LZB: & LinienZugBeeinflussung \\
\hline MRDSP: & Most Restrictive Dynamic Speed Profile \\
\hline MRSP: & Most Restrictive Speed Profile \\
\hline NEDAS: & Not-EDAS Driver Advisory System \\
\hline NP: & No Power state \\
\hline ODO: & ODOmetry \\
\hline RL: & Reference Location \\
\hline RTTP: & Real-Time Traffic Plan \\
\hline SB: & Stand-By state \\
\hline SIL: & Safety Integrity Level \\
\hline SF: & System Failure state \\
\hline SRS: & System Requirements Specification \\
\hline TIU: & Train Interface Unit \\
\hline TMS: & Traffic Management System \\
\hline TVM: & Transmission Voie-Machine \\
\hline UN: & Unfitted state \\
\hline VDAS: & Versatile Driver Advisory System. VDAS=EDAS+NEDAS \\
\hline
\end{tabular}

Some of these terms and abbreviations have been already defined in other documents (e.g. European Interoperability Directives; CENELEC documents, EEIG General Glossary)

\section{References}

[1] EPFL, DB Netz, TUD, UoNott, TRV (2013) Assessment of State-of-Art of Driver Advice Systems, Optimal Networks for Train Integration Management across Europe (ON-TIME), Task 6.1 (http://www.ontimeproject.eu/) 
[2] EPFL, Emery Daniel (2014), GLOSSARY-EDAS, FRS-EDAS, SRSEDAS-1-Introduction, SRS-EDAS-2-Description, SRS-EDAS-3-Principle, SRS-EDAS-3-States and Transitions, version 0.1

[3] Theeg G. et al. (2006), Comparison of international high speed signalling and control, Signal+Draht (98)

[4] UNott, (2013) Sample Human machine Interface (draft) - ON-TIME, D6.2 (draft)

[5] DB Netz (2012) DAS-Requirements_and_System_Designs - ON-TIME, WP 6 (internal document)

[6] ERA/ERTMS (2007), ERTMS/ETCS Functional Requirements Specification FRS, ERA/ERTMS/003204, http:/www.era.europa.eu/ Document-Register/Documents/Set-1-Index001-

ERA_ERTMS_003204\%20v500.pdf

[7] UNISIG (2000), SUBSET-023, SUBSET-026, Glossary and SRS, ERTMS/ETCS, http://www.era.europa.eu/Document-Register/Documents/ Set-1-Index003-SUBSET-023\%20v200.pdf; and http://www.era.europa.eu/ Document-Register/Pages/UNISIGSUBSET026.aspx

[8] ERA_ERTMS_015560 (2009), ETCS Driver Machine Interface, European Railway Agency

[9] EEIG-05E446 (2005), Operational DMI Information, ERTMS/ETCS Driver Machine Interface, Operational Rules Group

[10] EEIG-04E296 (2004), Operational DM Information, ERTMS Operational Rules Group, ERTMS Users Group 